\title{
THEORETICAL ANALYSIS OF FLUORESCENCE EXCITATION, ABSORPTION AND MULTIPHOTON-IONIZATION SPECTRA IN STRONG LIGHT FIELDS
}

\author{
Bernard DICK ${ }^{\ddagger}$ and R.M. HOCHSTRASSER \\ Department of Chemistry. Universitı of Pennsyluania. Philadelphia. Pennsylı ania 1910-1, USA
}

Recewed 12 Septe? ber 1983

\begin{abstract}
In a num'ser of experiments involving multiphoton extitaton with strong laser fields the ground state is coupled to one weitcd state - often bs a two-photon resonance - and also the higher excited states via additional one-photon resonances. In multiphoton-tonization experiments this second interaction can be used to induce the ionization. The Rabi frequency for this stiond interaction is often very large compared with the linewdth of the state of interest. In this letter the effect of thu strong interaction on the linesiapes of multiphoton ionization and fluorescence excitation spectra are described using an earlier developed mululevel multi-resonant model for the non-linear response in fields having arbitrary strengths. The revult predict broddening, iplitings and shifts of the line. in terms of the dynamical parameters of the system, and they predut effects not set observed.
\end{abstract}

\section{Introduction}

In recent years there were many new applications of moderately high-powered lasers in spectroscopic experiments. It is therefore of great interest to develop a detailed understanding of the effects of strong fields on the multulevel systems characteristic of molecular energy levels. In a previous paper [1] we presented detailed results of a calculdion of the reduced density matrix for a multilevel system interacting with strong fields. The off-diagonal elements of this density matrix give the "susceptihilities" for coherent light generation in these multilevel systems. The results can be applied to all the common non-linear spectrosecpies such as CARS. CSRS, coherent Rayleigh mixing. polarization spectroscopy and sum or difference frequency generation. However there are many common forms of spectroscopy that measure the consequences of the loss of macroscopic polarization rather than the coherently generated light. Examples of incoherent spectroscopies include absorption, fluorescence excitation. photoionization spectroscopy. and any form of chemical action spectroscopy. These spectroscopies can also be described in terms of the matrix elements of the reduced density matrix, and hence our method [1] can be used to study the strong field effects in incoherent spectroscopies as well. Both the off-diagonal (coherences) and the diugonal elements (populations) of the density matrix are needed in order to predict the form of the lineshapes and line positions ol such spectra under the influence of intense fields.

The purpose of the present note is to describe the spectra that are expected to arise in some rather common experimental situations, as schematically shown in fig. 1 . The system consists of three levels which could correspond to a set of mulecular electronic rovibrational states singled out of a more complex spectrum as a result of the choice of laser frequencies. A reservoir can be incorporated without changing the form of the results. The three arbitrarily spaced levels $\{a\rangle,|b\rangle$ and $|c\rangle$ are coupled through two interactions denoted in fig. 1 by $w_{1}$ and $w_{3}$, respectively. These interactions could involve one-photon resonant transitions, as assumed through-out in previous

\footnotetext{
" This research " as supported by a grant from the Narional Institutes of Hedth (GM-1 2592) and in part by NSF/MRL Program under Grant DNk-:2-16715.

"A rescarch Fellow ship af the "Deutsche lorschungsgemeinschatt" is pratefuliy acknowledged.
} 


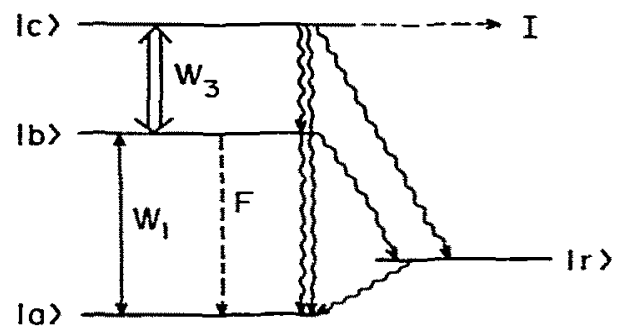

Fig. 1. Schematic representation of the three-level model used in this paper. $w_{3}$ and $w_{3}$ are the Rubi frequencies or their higher order equivalent for the weak and the strong interaction, respectively. A reservoir state in) which does not couple to any of the three states $(a),(b)$ or $(c)$ via the radiation field has no influence on the calculated lineshapes.

work ${ }^{*}$, but they could involve higher-order processes in general. The only requirement is that the coupling of levels $i$ and $j$ can be written in the form:

$$
\hat{w}=|j\rangle w_{i j} \exp (i \omega t)\langle i| \text {, }
$$

where the frequency $\omega$ is close enough to the resonance frequency $\omega_{i j}$ to validate the rotating wave approximation. For a one-photon resonant laser field we have, e.g.

$$
\vec{W}=|a\rangle \boldsymbol{\mu}_{a b} \cdot \varepsilon_{1} \exp \left(i \omega_{1} t\right)\langle b| \text {. }
$$

On the other hand, the interaction could be via a two-photon resonant combination of two fields $\varepsilon$ ' and ":":

$$
\hat{W^{\prime}}=|a\rangle \delta_{a b}: \varepsilon^{\prime} \varepsilon^{\prime \prime} \exp \left[i\left(\omega^{\prime}+\omega^{\prime \prime}\right) t\right]\langle b|,
$$

where $\boldsymbol{\delta}_{a b}$ represents a two-photon absorption tensor. Extension to even higher-order processes is straightforward. An interaction can be considered weak if the scalar factor $W$ in eq. (1), that is the resonant Rabi frequency $\boldsymbol{\mu} \cdot \varepsilon$ or its higher order equivalent $\delta: \varepsilon^{\prime} \varepsilon^{\prime \prime}$, etc., is smaller than the linewidth $\Gamma$ associated with the transition. This quantity may be kept small by restricting to the case of weak fields $\varepsilon$ and/or transition moment $\mu, \delta$ etc. In the case of two-photon absorption, for example, the magnitude of the two-photon transition tensor $\delta$ is often small enough for the interaction to betconsidered weak even when the fields $\varepsilon^{\prime}$ and $\varepsilon^{\prime \prime}$ are quite intense.

In the model discussed here $w_{1}$ is chosen as a weak interaction, while the $w_{3}$ interaction may be arbitrarily strong. This model corresponds closely to a photoionization experiment in which ions or electrons lisult from an upper state $(|c\rangle)$ which is excited by a strong laser field, while a weak field interaction excites the lower energy states: For example in a two-color two-photon resonant, three-photon ionization ${ }^{\text {t }}$. Naturally, questions arise regarding the effect of the strong field on the spectrum obtained by tuning the weaker one, and how the strong field affects the absorption or the fluorescence excitation spectrun. Furthermore in two-photon absorption situations there is often a $\mid b\} \rightarrow|c\rangle$ transition at the proper frequency to be driven by one of the fielas already present [3], and its effects should be carefully considered.

\section{Treatment of strong field effects}

In ref. [1] it was shown that the solution to the Liouville equation of motion for the density operator of an $N$. level system excited by as many as $N-1$ resonant sources can be written in the steady-state form:

$$
\rho(\infty)=x^{-1} a \text {. }
$$

Here $p(\infty)$ is the column vector of steady-state density matrix elements and $a$ is the equilibrium density matrix in the absence of the external fields. The matrix $X$, given explicitly in ref. [1], is a function of the damping param-

₹ The numbering scheme for the interaction has been adopted from our previous work as have been all other symbols describing dynamic parimeters of the system or the fields. In this nomenclature $\mid w_{3}$ couples levels $|b\rangle$ and $|c\rangle$, while the interaction coupling $|a\rangle$ and $|c\rangle$ is called $w_{2}$.

* We are not aware of such an experiment yet, but a two-color MPI experiment with time-separated pulses has been performed 121. 
eters $\Gamma_{i j}$ for level pairs separated by energy $\hbar\left(\omega_{i}-\omega_{j}\right)=\hbar \omega_{i j}$, the frequency mismatches between the exciting fields and the molecular transitions. the feeding parameters $\gamma_{i j}$ corresponding to the rate constants for populatior. iransfer betweer. molecular levels. and the field coupling factors $w_{n}(i \rightarrow j)$ where the field frequency $\omega_{n}$ is near resonant to the transition $i \rightarrow j$. The explicit form of $\mathbf{X}$ for the three-level case discussed here is:

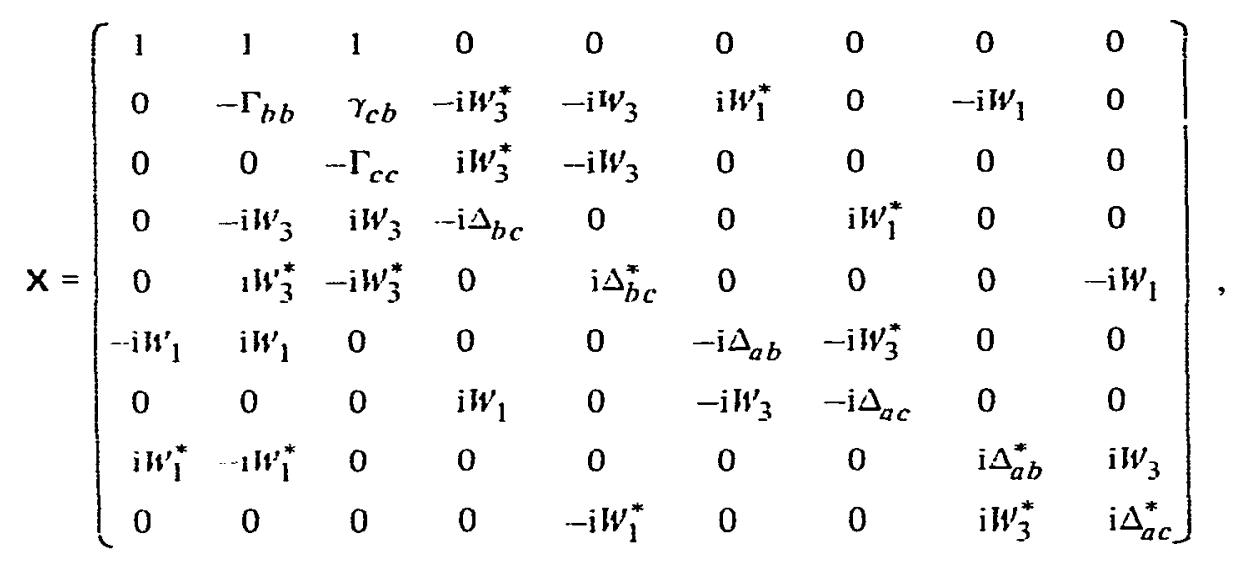

where $\lrcorner_{a b}=\omega_{a b}+\omega_{1}-\mathrm{i} \Gamma_{a b} \cdot \Delta_{b c}=\omega_{b c}+\omega_{3}-\mathrm{il}_{b c} \cdot \Delta_{a c}=\omega_{a c}+\omega_{1}+\omega_{3}-\mathrm{i} \Gamma_{a c} \cdot p(\infty)=\left(\rho_{a a} \cdot \rho_{b b} \cdot \rho_{c c} \cdot \rho_{b c}\right.$. $\left.\rho_{c b}, \rho_{a b}, \rho_{a c}, \rho_{b a}, \rho_{c a}\right)$.

Under the stated conditions. inversion of the $9 \times 9$ matrix $X$ will provide the diagonal and off-diagonal elements if the system density matrix for any ralues of $\mathrm{I}_{1}$ and $\mathrm{w}_{3}$. However. analytical solutions are readily obtained for the case that one or the other of these coupling strengths is small enough to allow a perturbative treatment.

In order to calculate the steady rate of a spontaneous process deriving from levels $|b\rangle$ or $|c\rangle$ we require to calauldte $\rho_{b b}$, and $\rho_{c c}$. In the case of ionization of state $|c\rangle$ we ascribe a rate constant $\gamma_{I}$ to this process, and assume that the svstem population $T_{r} \rho$ is not significantly altered by this decay. The form of the multiphoton-ionization spectrum. $I$. is then given by

$$
l\left(\omega_{1} \cdot \omega_{j}\right)=\gamma_{I} \rho_{1} c
$$

In $a^{n}$ analogous manner the fluorestence excitation spectrum, $F$, of the $a \rightarrow b$ transition with the radiative rate constant $\gamma_{\mathrm{T} b}$ for state $|b\rangle$ is:

$$
F\left(\omega_{1}, \omega_{3}\right)=\gamma_{\mathrm{r} b}, \rho_{b b} .
$$

This result is exact if the fluorescence is detected wide-band (i.e. integrated over all frequencies). otherwise disInctions between fluorescence and Raman would need to be made [4]. These results are appropriate for either beam or bulb experiments. Both photoionization and fluorescence excitation spectra are usually regarded as a measure of the absorption spectrum $A$, which has the form $[5.6]$ :

$$
A\left(\omega_{1}, \omega_{3}\right)=\left\langle\dot{P}\left(\omega_{1}\right) \epsilon_{1}\left(\omega_{1}\right)\right\rangle=\operatorname{im}\left[\rho_{a b}\right],
$$

where $P\left(\omega_{1}\right)$ is the macroscopic polarization induced in the medium by the applied fields. This form is also appropriate for the case of two-photon absorption using the fields in the general form given in eq. (3). In the following we obtain explicit formulas for the three types of spectra described by eqs. (6)-(8).

Thro damping parameters $\Gamma_{i j}$ which appear in the theory have the form:

$$
\Gamma_{i j}=\frac{1}{2}\left(\Gamma_{i}+\Gamma_{j}\right)+\Gamma_{i j}^{\prime},
$$

where $\Gamma_{i}$ as.d $\Gamma_{j}$ are the population decay rates. and $\Gamma_{i j}^{\prime}$ is the rate of pure dephasing. The pure dephasing which can be a rest. It of collisions in the impact limit is zero for beam conditions, in which case some of the formulas 
are simplified because any two of the transition linewidths in the three level system, $\Gamma_{a b}, \Gamma_{a c}$ or $\Gamma_{b c}$, then determine the third one.

\section{Results}

We treat $W_{3}$ as the strong field in (5) and expand the solution of (4) in powers of the weak interaction $w_{1}$. This yields in first order (eq. (71a,d) of ref. [1]):

$$
\rho_{a b}=-\frac{w_{1} \Delta_{a c}}{\Delta_{a b} \Delta_{a c}-w_{3}^{2}} ; \quad \rho_{a c}=\frac{w_{1} w_{3}}{\Delta_{a b} \Delta_{a c}-w_{3}^{2}} .
$$

The populations are obtained from the $5 \times 5$ equation system of the second order problem. This yields for $\rho_{c c}$ and $\rho_{b b}$ :

$$
\begin{aligned}
\rho_{c c} & =\frac{w_{1}^{2} w_{3}^{2} 2 / \Gamma_{c c}}{\Delta_{b c} \Delta_{b c}^{*}+2 g \Gamma_{b c} w_{3}^{2}} \operatorname{Im}\left(\frac{\left(2 \Gamma_{b c} / \Gamma_{b b}\right) \Delta_{a c}-\Delta_{b c}^{*}}{\Delta_{a b} \Delta_{a c}-W_{3}^{2}}\right) \\
\rho_{b b} & =\frac{2 w_{1}^{2}}{\Gamma_{b b}} \operatorname{Im}\left(\frac{\Delta_{a c}}{\Delta_{a b} \Delta_{a c}-W_{3}^{2}}\right)+\frac{\gamma_{c b}-\Gamma_{c c}}{\Gamma_{b b}} p_{c c},
\end{aligned}
$$

with $g=\left(\Gamma_{b b}+\Gamma_{c c}-\gamma_{c b}\right) /\left(\Gamma_{c c} \Gamma_{b b}\right)$. According to eqs. (6)-(8), $\rho_{a b}$ determines the absorption spectrum, $\rho_{c c}$ the photoionization spectrum and $\rho_{b b}$ the fluorescence excitation spectrum. All three spectra contain the resonance:

$$
\operatorname{Im}\left(\frac{\Delta_{a c}}{\Delta_{a b} \Delta_{a c}-w_{3}^{2}}\right) \stackrel{w_{3} \rightarrow 0}{\longrightarrow} \operatorname{Im}\left(1 / \Delta_{a b}\right)=\frac{\Gamma_{a b}}{\left(\omega_{a b}+\omega_{1}\right)^{2}+\Gamma_{a b}^{2}}
$$

which reduces to a simple lorentzian in the limit of weak $W_{3}$. If all the population of level $|c\rangle$ decays only into level $|b\rangle$, which means $\gamma_{c b}=\Gamma_{c c}$, then the fluorescence excitation spectrum is identical with the direct absorption spectrum. The origin of the extra term that occurs when $\gamma_{c b} \neq \Gamma_{c c}$ is readily seen from the expansion of $\rho_{c c}$ in powers of $w_{3}$. The lowest order factor is the fourth-order population term $\rho_{c c}^{(4)}$ of the conventional perturbation theory treatment [7-9] of the Liouville equation:

$$
\begin{aligned}
\rho_{c c}^{(4)} & =\frac{4 W_{1}^{2} w^{\frac{2}{3}}}{\Gamma_{c c} \Gamma_{b b}} \operatorname{Im}\left(1 / \Delta_{b c}\right) \operatorname{Im}\left(1 / \Delta_{a b}\right) \\
& -\frac{2 W_{1}^{2} w^{\frac{2}{3}}}{\Gamma_{c c}} \operatorname{Im}\left(1 / \Delta_{a b} \Delta_{a c} \Delta_{b c}\right) .
\end{aligned}
$$

The part (14a) describes a stepwise excitation, leading first to a population of level $|b\rangle$ (the $1 / \Delta_{a b}$ resonance) which is consequently excited to a level $|c\rangle$ (the $1 / \Delta_{b c}$ resonance). In contrast (14b) does not involve a population of level $|b\rangle$, but describes a direct two-quantum excitation of level $|c\rangle$ through simultaneous absorption of one quantum from each of the $w_{1}$ and the $w_{3}$ fields. For example, if the $w_{1}$ field describes a two-photon type interaction, eq. (14b) accounts for the direct three-photon excitation of level $|c\rangle$. Obviously this leads to an additional resonance if $\omega_{3}$ is fixed detuned by an amount $d$ from the $\omega_{c b}$ resonance. The ordinary resonance occurs at $\omega_{1}$ $=\omega_{b a}$ independent of the detuning while the double-quantum resonance peaks at $\omega_{1}=\omega_{c a}-\omega_{3}=\omega_{b a}+a$. 


\section{Simulations of spectra and predictions}

The spectra for various common experimental conditions are readily obtained from eqs. (10)-(12). The parameter values are chusen to represent molecular or atomic systems that are likely to be encountered in realistic experimental situations.

The largest variety of spectral lineshapes and resonances is expected when both excited levels $(b)$ and $(c)$ have small Inewidths compared with the Rabi frequency $\boldsymbol{w}_{3}$ - An example of such a situation could be an atomic system with the level $|c\rangle$ just below the ionization energy. Ionization of atoms excited to level $|c\rangle$ can then be achieved though a pulsed dc electric field after the excitation. under which conditions sharp-line spectra for the level $|c\rangle$ can be obtained by monitoring the photoelectron current [10-13]. Fig. 2 shows typical lineshapes expected in such a situation, if the $\mid \psi_{3}$ field is fixed on the $|b\rangle \rightarrow|c\rangle$ resonance. and $\omega_{1}$ is tuned over the $|a\rangle \rightarrow|b\rangle$ resonance. The choice of the decay parameters $\Gamma$ and $\gamma$ correspond to the case of no pure dephasing, this means collisionless conditions in the gas phase or very low temperatures in solids. For a very weak $W_{3}$ field a single lorentzian line having the width $\Gamma_{a b}$ is found in the absorption and fluorescence excitation spectra. The photoionization spectrum in this limit is considerably narrower in the wings, obviously as a consequence of interference between non-linear processes involving the population of level $|b\rangle$ and the direct two-quantum excitation of level $|c\rangle$. With increasing $w_{3}$ the line i-roadens and fually splits symmetrically into two lines. This splitting occurs at higher field strengths in photoionization than in fluorescence excitation (fig. 2). In the limit of a very strong $\boldsymbol{w}_{3}$ field, $\boldsymbol{w}_{3} \gg \Gamma_{a b}, \Gamma_{a c}$, the spectrum consists of two nearly lorentzian lines separated by $2 W_{3}$ and both having a width of the average of the $|a\rangle \rightarrow|b\rangle$ and $|a\rangle \rightarrow|c\rangle$ transitions (last spectrum in figs. 2a and $2 b$ ).

A somewhut different situation arises when the stror: 1 eld $\boldsymbol{w}_{3}$ is fixed but detuned from the $|b\rangle \rightarrow|c\rangle$ resonance by a frequency $d$. The spectra expected in this case are simulated in fig. 3 with the same dynamical parameters used for fig. 2. The splitting of the signal is now unsymmetrical: while the line at $\omega_{1}=\omega_{b a}$ shifts, a new line evolves at $\omega_{1}=\omega_{b a}-d$ for absorption and fluorescence excitation (fig. $3 b$ ). This second line rapidly grows in intensity with increasing $w_{3}$. and in the limit of large $w_{3}$ the two line maxima are separated by $2 w_{3}+d$. While it shows the same behaviour as the absorption and fluorescence excitation spectra for large $w_{3}$. the photoionization spectrum differs in the limit of small $\boldsymbol{u}_{3}^{\prime}$. since the resonance at $\omega_{1}=\omega_{b a}-d$ is always present. The origin of this resonance corresponds to the situation $\omega_{1}+\omega_{3}=\omega_{c o}$ and is. therefore. related to the direct two-quantum excitation of level $|c\rangle$.
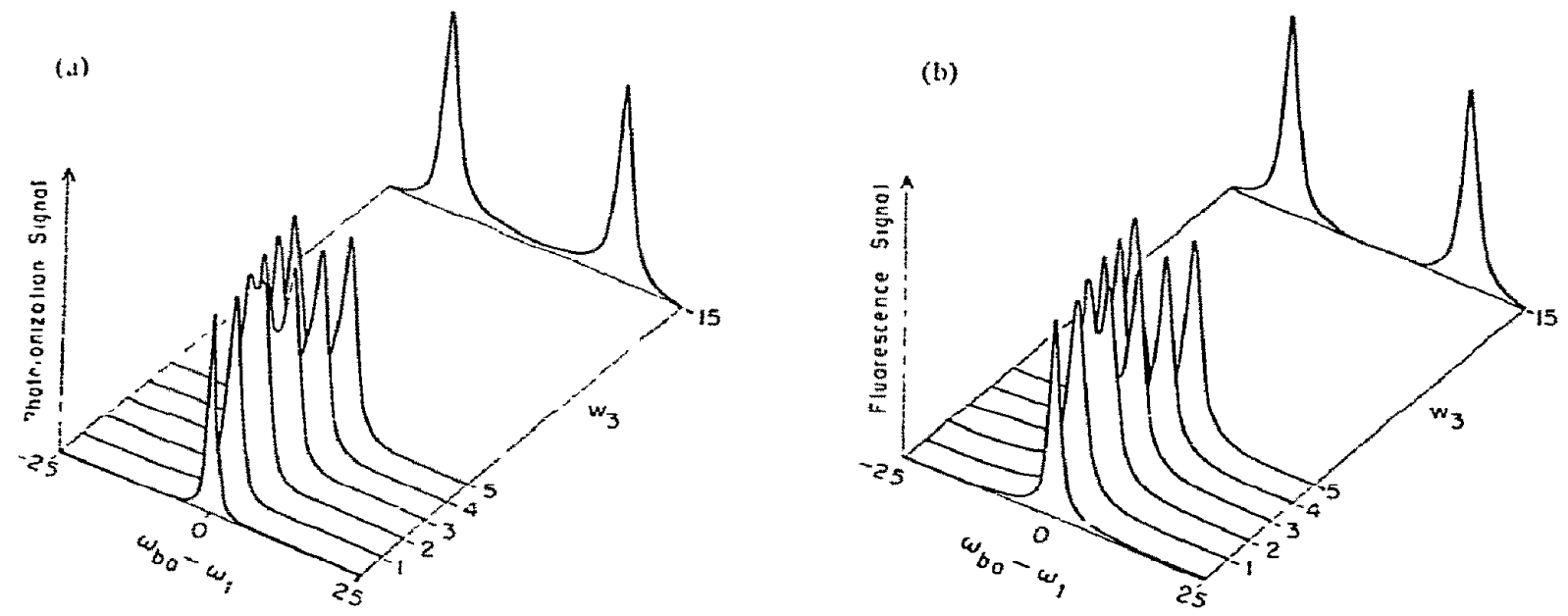

1ํ. 2. Simulition of lineshapes with the strong field fived on resonance. (a) photoionication spectrum, (b) thorescence excitation spectra. The parameters chosen for this simulation are: $r_{a b}=1, \Gamma_{a c}=2 . r_{b c}=3 . \gamma_{b a}=\gamma_{c a}=\gamma_{c b}=2$, corresponding to pure radiative damping. All spectra hase been sealed to their maximum values. 

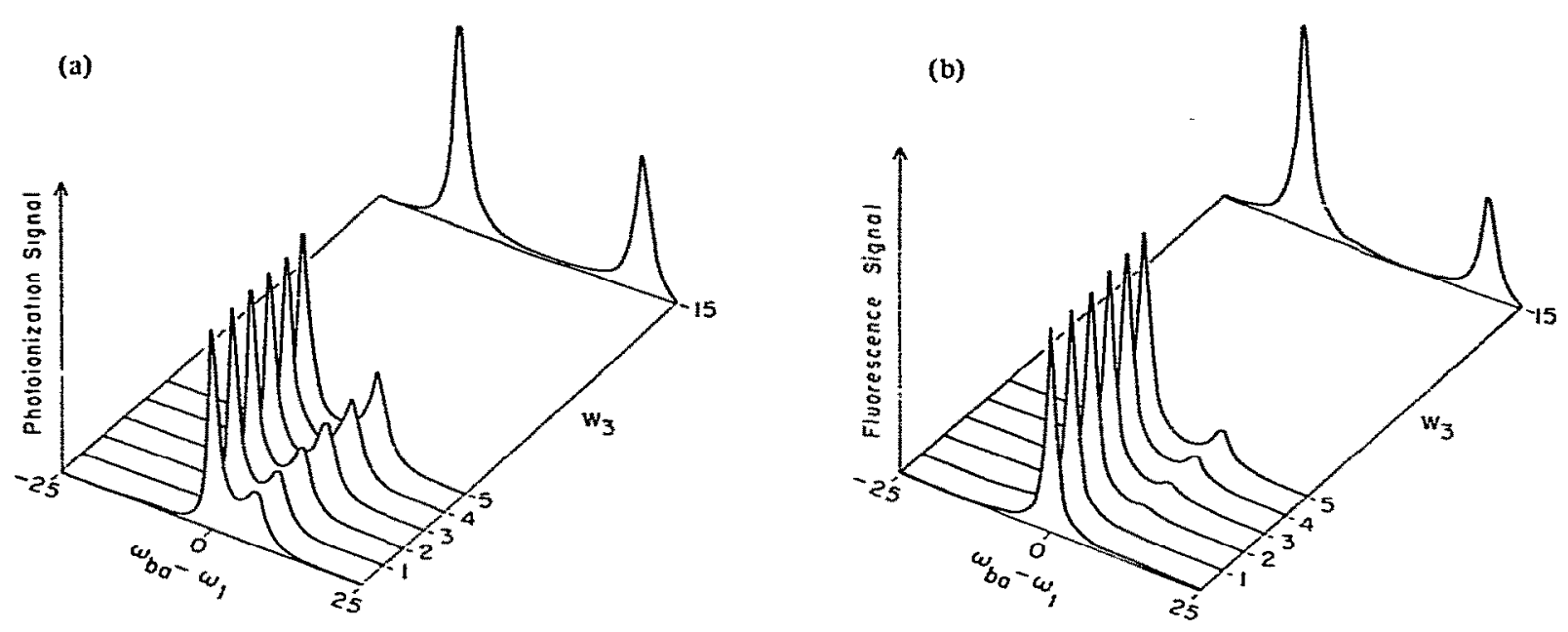

rig. 3. Simulation of lineshapes with the strong field detuned from the $(b)-|c\rangle$ resonance. (a) photoionization spectra. (b) fluorescence excitation spectra. Parameters are the same as for fig. 2 , with the detuning set to $d=8$.

In an expansion in powers of $w_{3}$ these terms are contained in the photoionization expression already in the lowest order while corresponding processes in absorption or fluorescence excitation appear only in higher orders.

Another situation more often encountered in experiments on molecular systems is described by a model which contains a sharp $|a\rangle \rightarrow|b\rangle$ transition, but a very broad $|a\rangle \rightarrow|c\rangle$ transition $\left(\Gamma_{a b} \ll \Gamma_{a c}\right)$. This will naturally be the case if $|c\rangle$ is an autoionizing level embedded in a continuum. Values of $w_{3}$ achievable with conventional dye lasers could be larger than $\Gamma_{a b}$, but could be much smaller than $\Gamma_{a c}$. A splitting of the various signals can therefore not be produced, and the two-quantum contribution to the lineshape will become negligible. Absorption, fluorescence excitation and photoionization will, therefore, yield the same spectral lineshapes. Some simulations for such a case are shown in fig. 4 . For a weak $w_{3}$ field a resonance with essentially the natural linewidth $\Gamma_{a b}$ of the $\mid a$ ) $\rightarrow|b\rangle$ transition is observed. With increasing $W_{3}$ this line is broadened to

$$
\widetilde{\Gamma}=\Gamma_{a b}+w \frac{2}{3} / \Gamma_{a c} \text {. }
$$

but remains essentially lorentzian. If $W_{3}$ represents a one-photon resonant interaction, the line-broadening will vary linearly with the intensity of the $\omega_{3}$ source and scale with the oscillator strength of the $b \rightarrow c$ transition. A plot of

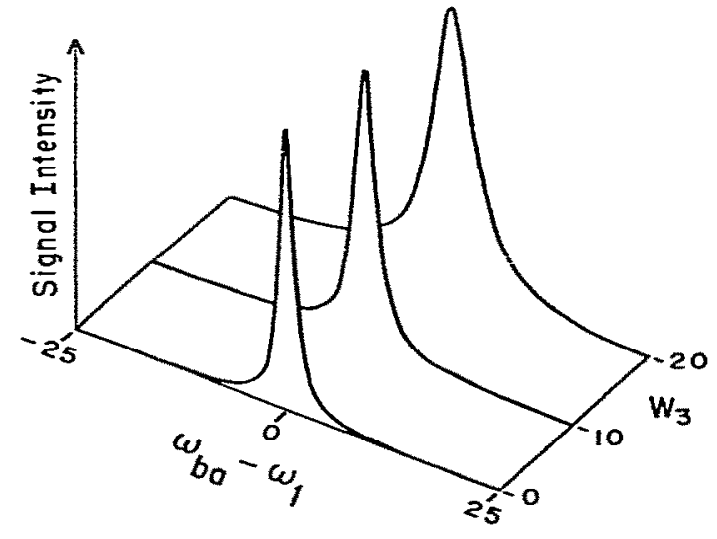

Fig. 4. Simulation of lineshapes for the case of a very-shortlived final state $(c)$. Parameters are: $\Sigma_{a b}=1, \Gamma_{a c}=100, \gamma b a$ $=2, \gamma_{c a}=200$ and no pure dephasing. The detuning of the strong field from the $|b\rangle \rightarrow|c\rangle$ resonance was set $10 d=100$. 
the observed linewidth $\widetilde{\Gamma}$ versus the intensity of the $W_{3}$ field should yield the molecular parameters $\Gamma_{a b}$ and $\mu_{b}^{2} / \Gamma_{a c}$. This could give information about linewidths of transitions in the far VuV region and dipoles of excited state transitions not easily accessible by direct absorption measurements.

A detuning of $\omega_{3}$ from resonance merely results in a shift of the resonance with increasing $w_{3}$. This shift should only be detectable if the detuning is on the order of the linewidth of the broad state $|c\rangle$. If the photoionizing level $(c)$ is sufficiently broad it makes no difference whether $\omega_{3}$ is fixed or is tuned over a small range corresponding to the linewidth of level $|b\rangle$. The spectra in fig. 4 are. therefore. also appropriate. when both interactions $w_{1}$ and $w_{3}$ result from the same laser. An example for this situation is the two-photon resonant three-photon ionization with a single laser. In this case $\omega_{c b} \approx \frac{1}{2} \omega_{b a}$. Two $\omega_{1}$ photons cause the $a \rightarrow b$ transition. and one $\omega_{1}$ photon causes the photoionization.

\section{Conclusions}

The purpose of this paper was to show that common forms of spectroscopy. such as fluorescence excitation. absorption or photoionization. can be modelled even in the presence of strong fields. A matrix $(X)$ is given which can be used to predict these spectra in the steady-state approximation. As an example of the use of this time-independent form of the Liouville equation the spectra of a three-level system were obtained explicitly for the case of twocolor resonant excitation with one of the light sources being weak. The absorption, photoionization and fluorescence excitation spectra can be different depending on conditions of laser power and detuning from resonances. In the cise where the photoionizing state (or more generally, photochemically active state) is diffuse, having a lifetime less than the Ribi times of the fields, then the spectra are all the same and simply broaden with increasing laser power. These same rechniques can be used to study spectra in four-level systems using the $16 \times 16$ matrix in our previous paper [1].

\section{References}

[1] B. Dick and R.M Hoinstrasser, Chem. Phys. 75 (1983) 133.

[2] U. Bues. H.I. Neuser and E.W. Sehles. J. Chem. Whys. 72 (1980) 4327.

131 B. Dick. H. Gonsha and G. Hohlneicher. Ber. Bunsenges. Mhy sik. Chem. 85 (1981) 7.76 .

H H H.R Mollow, Phys. Rev. 188 (1969) 1969.

[5] A Blocmuergen. Nonlutar optics (Benjamin, New York, 1965).

[6] Y R. Shen. Phys. Rev. 139 (1974) 622

17] S.Y. Lee. T.K. Gustation. S.A.J. Donet and J.P.E. Taran, Opt. Commun. 23 (1977) 1.

[S] $T K$ Yee and $T K$ Gustafion, Phys Rev. A18 (1978) 1597.

19] J.-1. Oudar and Y.R. Shen, Phys. Rev. 122 (1980) 1141.

(10) C. Fabre, P. Goy and S Haroche. J. Phys. B10 (1977) 1183.

$111]$ T.F Gallagher, L M. Humphrey. R.M. Hill. W.t. Cooke and S A. Edelstein. Phy s. Rev. A15 (1977) 1937.

[12] K.C llarney and B.P. Stoichetf. Phys. Rev. Letters 38 (1977) 537 .

[13] G. Leuchs and II. Wather. Z. Physik A293 (1979) 93. 